\title{
QUASI-NILPOTENT SETS IN SEMIGROUPS
}

\author{
H. L. CHOW
}

ABSTRACT. In a compact semigroup $S$ with zero 0 , a subset $A$ of $S$ is called quasienilpotent if the closed semigroup generated by $A$ contains 0 . A probability measure $\mu$ on $S$ is called nilpotent if the sequence $\left(\mu^{n}\right)$ converges to the Dirac measure at 0 . It is shown that a probability measure is nilpotent if and only if its support is quasi-nilpotent. Consequently, the set of all nilpotent measures on $S$ is convex and everywhere dense in the set of all probability measures on $S$ and the union of their supports is $S$.

In a topological semigroup with zero 0 , an element $x$ is termed nilpotent if $x^{n} \rightarrow 0$ as $n \rightarrow \infty[5]$. This definition has an obvious extension to subsets of the semigroup, i.e. a subset $A$ is nilpotent if $A^{n} \rightarrow 0$ as $n \rightarrow \infty$. Now we call a subset $B$ of the semigroup quasi-nilpotent if the closed semigroup generated by $B$ contains the zeio 0 . It is shown that, when the topological semigroup is compact, a singleton is nilpotent if and only if quasinilpotent. Then we investigate the set of probability measures on a compact semigroup and characterize a nilpotent probability measure as a measure with quasi-nilpotent support.

Let $S$ be a topological semigroup with zero 0 , and $A$ a subset of $S$. Let $S(A)$ denote the semigroup generated by $A$, i.e. $S(A)=\bigcup_{n=1}^{\infty} A^{n}$. It is trivial that any subset containing 0 is quasi-nilpotent; in particular, the set $N(S)$ of nilpotent elements of $S$ is quasi-nilpotent. From the semigroup $S$ given in Example 6 below, in which $N(S)=[0,1)$ and $N(S)^{n}=N(S)$ for all $n[4$, p. 56], we see that $N(S)$ is not nilpotent.

Theorem 1. Let $A$ be a subset of $S$. Then (i) If $\overline{S(A)} \cap N(S) \neq \varnothing$ (where the bar denotes closure), then $A$ is quasi-nilpotent.

(ii) If $A^{n}$ is quasi-nilpotent for some $n$, then $A$ itself is quasi-nilpotent.

Proof. (i) Take $a \in \overline{S(A)} \cap N(S)$. In view of the fact that $a^{n} \rightarrow 0$, we have $0 \in \overline{S(A)}$, i.e. $A$ is quasi-nilpotent.

(ii) Since $S\left(A^{n}\right) \subset S(A)$ and $0 \in \overline{S\left(A^{n}\right)}$, it follows that $0 \in \overline{S(A)}$, and the theorem is proved.

We remark that, if $A^{n}$ is nilpotent for some $n$, then $A$ is also nilpotent, by a similar argument to that given in the proof of Lemma 2.1.4 of [4].

Received by the editors April 3, 1974 and, in revised form, July 15, 1974.

AMS (MOS) subject classifications (1970). Primary 22A20, 43A05, 60B15; Secondary 22A 15.

Key words and phrases. Quasi-nilpotent set, compact semigroup with zero, probability measure, support of a measure, nilpotent measure, nil semigroup. 
Evidently a nilpotent set is quasi-nilpotent. As for the converse, which may not be true in general, we prove a special case in

Theorem 2. Suppose $S$ is a compact semigroup with 0 . Then $x \in S$ is nilpotent if and only if quasi-nilpotent.

Proof. It is enough to show that $x$ is nilpotent if it is quasi-nilpotent. Recall that the minimal ideal $K(\overline{S(x)})$ of the compact semigroup $\overline{S(x)}$ contains exactly all cluster points of the sequence $\left(x^{n}\right)_{n=1}^{\infty}$ (see, for example, [4, Theorem 3.1.1]). Now $K(\overline{S(x))}=\{0\}$ since $0 \in \overline{S(x)}$. Thus the sequence $\left(x^{n}\right)$ has a unique cluster point, whence $x^{n} \rightarrow 0$ as $n \rightarrow \infty$, completing the proof.

Remark. The preceding theorem does not hold for a compact semitopological semigroup (i.e. the multiplication is only separately continuous). For instance, take the compact monothetic semigroup $S_{w}(\mu)$ generated by $u$, with $\mu$ defined in Example 2 of [1]; then the semigroup has zero 0 and identity 1 such that $u^{n ! / 2} \rightarrow 0$ and $u^{n !} \rightarrow 1$. As a consequence, the element $u$ is quasi-nilpotent but not nilpotent.

In what follows $S$ will be a compact semigroup with zero 0 。 Denote by $P(S)$ the set of probability measures (i.e. normalized positive regular Borel measures) on $S$. For $\mu, \nu \in P(S)$, define convolution $\mu \nu \in P(S)$ by

$$
\int f(z) d \mu \nu(z)=\iint f(x y) d \mu(x) d \nu(y)
$$

for all continuous functions $f$ on $S$, so that $P(S)$ forms a semigroup. If $P(S)$ is endowed with the weak ${ }^{*}$ topology, i.e. a net $\left(\mu_{\alpha}\right)$ in $P(S)$ converges to $\mu \in P(S)$ if $\int f(x) d \mu_{\alpha}(x) \rightarrow \int f(x) d \mu(x)$ for continuous functions $f$ on $S$, then $P(S)$ is a compact semigroup [3].

The support of $\mu \in P(S)$, supp $\mu$, is the smallest closed set with $\mu$-mass 1. It is well known [3, Lemma 2.1] that, for $\mu, \nu \in P(S)$, supp $(\mu \nu)=(\operatorname{supp} \mu)$ - $(\operatorname{supp} \nu)$.

Let $\Gamma$ be a subset of $P(S)$ and define its support as the set supp $\Gamma=$ $\overline{U_{\mu \epsilon \Gamma} \operatorname{supp} \mu}$. It is easy to see that $\operatorname{supp}\left(\Gamma_{1} \Gamma_{2}\right)=\left(\operatorname{supp} \Gamma_{1}\right)\left(\operatorname{supp} \Gamma_{2}\right)$ for $\Gamma_{1} \subset P(S), \Gamma_{2} \subset P(S)$.

Lemma 3. Let $\Gamma \subset P(S)$. Then supp $\overline{S(\Gamma)}=\operatorname{supp} S(\Gamma)=\overline{S(\operatorname{supp} \Gamma)}$.

Proof. That supp $\overline{S(\Gamma)}=\operatorname{supp} S(\Gamma)$ follows from a result in [3, p. 55]. We assert that supp $S(\Gamma)=\overline{S(\operatorname{supp} \Gamma)}$. Since $S(\Gamma) \supset \Gamma^{n}$ for $n=1,2, \ldots$, clearly supp $S(\Gamma) \supset$ supp $\Gamma^{n}=(\operatorname{supp} \Gamma)^{n}$ and so supp $S(\Gamma) \supset S(\operatorname{supp} \Gamma)$.

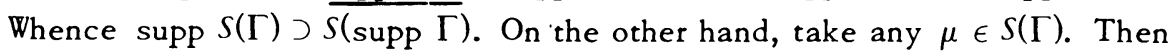
$\mu \in \Gamma^{n}$ for some $n$, implying that supp $\mu \subset \operatorname{supp} \Gamma^{n}=(\operatorname{supp} \Gamma)^{n} \subset S(\operatorname{supp} \Gamma) \subset$

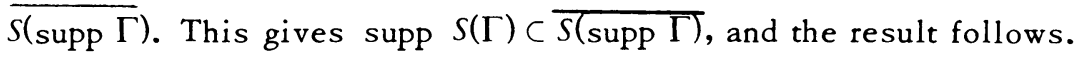

Since the Dirac measure $\theta$ at 0 is a zero in $P(S)$, we can now consider quasi-nilpotent sets in $P(S)$. 
Theorem 4. A subset $\Gamma \subset P(S)$ is quasi-nilpotent if and only if supp $\Gamma$ is quasi-nilpotent in $S$.

Proof. Suppose first that $\Gamma$ is quasi-nilpotent, i.e. $\theta \in \overline{S(\Gamma)}$. By virtue of Lemma 3, we have $0 \in \overline{S(\operatorname{supp} \Gamma)}$ i.e. supp $\Gamma$ is quasi-nilpotent. Conversely, suppose supp $\Gamma$ is quasi-nilpotent in $S$. This means that $0 \epsilon$ $\overline{S(\operatorname{supp} \Gamma)}$ and therefore $\{0\}$ is the minimal ideal $K \overline{(S(\operatorname{supp} \Gamma))}$ of the semigroup $\overline{S(\operatorname{supp} \Gamma)}$. Now consider the minimal ideal $K \overline{(S(\Gamma))}$ of the compact semigroup $\overline{S(\Gamma)}[6$, Theorem 2]. Since supp $K(\overline{S(\Gamma))}=K($ supp $\overline{S(\Gamma)})$ (see, for example, [2, Theorem 5(2)]) and supp $\overline{S(\Gamma)}=\overline{S(\operatorname{supp} \Gamma)}$ by Lemma 3, we have $\{0\}=\operatorname{supp} K \overline{(S(\Gamma))}$, giving that $K(\overline{S(\Gamma)})=\{\theta\}$. Accordingly $\theta \in \overline{S(\Gamma)}$, and the theorem is proved.

By Theorems 2 and 4, we immediately obtain

Theorem 5. A measure $\mu \in P(S)$ is nilpotent if and only if supp $\mu$ is quasi-nilpotent in $S$.

Example 6. The result in Theorem 5 is best possible in the sense that the support of a nilpotent measure in $P(S)$ need not be a nilpotent subset of $S$. Take the semigroup $S=[0,1]$ with the usual topology and the ordinary multiplication. Let $\mu$ be the restriction to $S$ of the Lebesgue measure on the real line. Since supp $\mu=S$ is quasi-nilpotent, it follows that $\mu$ is nilpotent. However, supp $\mu$ is not nilpotent since $(\operatorname{supp} \mu)^{n}=\operatorname{supp} \mu=S$ for all $n$.

Note that Theorem 5 is not true for the compact semitopological semigroup $S_{w}(\mu)$ considered in the Remark above. Obviously the Dirac measure $\delta(u)$ at $u$ is not nilpotent while supp $\delta(u)$ is quasi-nilpotent in $S$ 。

Applying Theorem 5, we obtain the following results about the set $N(P(S))$ of nilpotent elements in $P(S)$. First we have a sufficient condition for a probability measure to be nilpotent.

Theorem 7. Let $\mu \in P(S)$. If supp $\mu \cap N(S) \neq \varnothing$, then $\mu \in N(P(S))$.

Proof. Since $\overline{S(\operatorname{supp} \mu)} \cap N(S) \supset$ supp $\mu \cap N(S) \neq \varnothing$, we see that the set supp $\mu$ is quasi-nilpotent in $S$ by Theorem 1 (i). Whence $\mu$ is nilpotent.

Example 8. The converse of Theorem 7 may not hold. For instance, take the semigroup $S$ with the following multiplication table:

\begin{tabular}{l|llll} 
& 0 & $a$ & $b$ & $c$ \\
\hline 0 & 0 & 0 & 0 & 0 \\
$a$ & 0 & 0 & $a$ & 0 \\
$b$ & 0 & 0 & $b$ & 0 \\
$c$ & 0 & $a$ & $a$ & $c$
\end{tabular}

Then the measure $\mu=1 / 2(\delta(b)+\delta(c)) \in N(P(S))$ since $0 \in$ supp $\mu^{2}$. However, $\operatorname{supp} \mu \cap N(S)=\{b, c\} \cap\{0, a\}=\varnothing$. 
Corollary 9。(i) $N(P(S))$ is a noncountable set.

(ii) $\bigcup$ supp $\mu: \mu \in N(P(S))\}=S$.

Proof. (i) Take any measure $\mu \neq \theta$ and real number $0 \leq t<1$. Then the measure $t \mu+(1-t) \theta$ is nilpotent since $0 \in \operatorname{supp}(t \mu+(1-t) \theta) \cap N(S)$. Hence the set $N(P(S)) \supset\{t \mu+(1-t) \theta: 0 \leq t<1\}$ and so is noncountable.

(ii) Let $a \in S$. Since $0 \in \operatorname{supp} 1 / 2(\delta(a)+\theta) \cap N(S)$, it follows that $1 / 2(\delta(a)+\theta) \in N(P(S))$. That $a \in \operatorname{supp} 1 / 2(\delta(a)+\theta)$ gives the result.

A semigroup with zero is said to be nil if each element is nilpotent.

Theorem 10. $P(S)$ is nil if and only if $S$ is nil.

Proof. The "if" part follows from the fact that, for $\mu \in P(S)$, supp $\mu$ $\cap N(S)=\operatorname{supp} \mu \neq \varnothing$. To prove the "only if" part, take $a \in S$ and note that $\delta(a)$ is nilpotent in $P(S)$. So $a$ is nilpotent in $S$ and the proof is complete.

Lemma 11. Let $\mu, \nu \in P(S)$. If $\mu \in N(P(S))$ and supp $\mu \subset \operatorname{supp} \nu$, then $\nu \in N(P(S))$.

Proof. This is immediate since $0 \in \overline{S(\operatorname{supp} \mu)} \subset \overline{S(\operatorname{supp} \nu)}$.

Theorem 12. (i) $N(P(S))$ is a convex set and hence connected.

(ii) $\overline{N(P(S))}=P(S)$.

Proof。(i) Take $\mu, \nu \in N(P(S))$. For real number $0<t<1$, the.measure $t \mu+(1-t) \nu \in N(P(S))$ since

$$
\operatorname{supp}(t \mu+(1-t) \nu)=\operatorname{supp} \mu \cup \operatorname{supp} \nu \supset \operatorname{supp} \mu .
$$

Thus $N(P(S))$ is convex.

(ii) Let $\tau \in P(S)$. Clearly $\theta / n+(n-1) \tau / n \in N(P(S))$ for any positive integer $n$. As the sequence $\left(\theta / n+(n-1)_{\tau} / n\right)_{n=1}^{\infty}$ converges to $\tau$, we see that $N(P(S))$ is dense in $P(S)$.

Corollary 13. Let $W$ be a subset of $P(S)$. If $W \supset N(P(S))$, then $W$ is a connected set.

Proof. This follows simply from the previous theorem.

For any $\mu \in P(S)$, it is a well-known fact that the sequence $\left(\left(\mu+\mu^{2}+\cdots+\mu^{n}\right) / n\right)_{n=1}^{\infty}$ must converge to a measure $L(\mu) \in P(S)$ such that supp $L(\mu)$ is the minimal ideal of the semigroup $\overline{S(\operatorname{supp} \mu)}$; see [7] or [8].

Theorem 14. The measure $\mu \in P(S)$ is nilpotent if and oniy if $L(\mu)=\theta$.

Proof. In view of the fact that $L(\mu)=\theta$ if and only if $\overline{S(\operatorname{supp} \mu)}$ contains 0 , we apply Theorem 5 to conclude the proof. 


\section{REFERENCES}

1. G. Brown and W. Moran, Idempotents of compact monothetic semigroups, Proc. London Math. Soc. (3) 22 (1971), 203-216. MR 44 \#5408.

2. H. L. Chow, $O_{n}$ supports of semigroups of measures, Proc. Edinburgh Math. Soc. (2) 19 (1974), 31-33.

3. I. Glicksberg, Convolution semigroups of measures, Pacific J. Math. 9 (1959), 51-67. MR $21 \# 7405$.

4. A. B. Paalman-de Miranda, Topological semigroups, 2nd ed., Math. Centre Tracts 11, Mathematisch Centrum, Amsterdam, 1970.

5. K. Numakura, On bicompact semigroups with zero, Bull. Yamagata Univ. (Nat. Sci.) 1951, no. 4, 405-412. MR 16, 447.

108. MR 14, 18.

6. - On bicompact semigroups, Math. J. Okayama Univ. 1 (1952), 99-

7. M. Rosenblatt, Limits of convolution sequences of measures on a compact topological semigroup, J. Math. Mech 9 (1960), 293-305; addendum, ibid. 10 (1961), 681. MR 22 \#9544; 22, p. 2547.

8. S. Schwarz, Convolution semigroup of measures on compact non-commutative semigroups, Czechoslovak Math. J. 14 (89) (1964), 95-115. MR 30 \#212.

DEPARTMENT OF MATHEMATICS, CHUNG CHI COLLEGE, THE CHINESE UNIVERSITY OF HONG KONG, HONG KONG 